University of Nebraska - Lincoln

DigitalCommons@University of Nebraska - Lincoln

\title{
$9-2014$
}

\section{Implementation and Validation of Aortic Remodeling in Hypertensive Rats}

\author{
Shijia Zhao \\ University of Nebraska-Lincoln, s-szhao3@unl.edu \\ Linxia Gu \\ University of Nebraska-Lincoln, gul@fit.edu
}

Follow this and additional works at: https://digitalcommons.unl.edu/mechengfacpub

Part of the Biomedical Devices and Instrumentation Commons

Zhao, Shijia and Gu, Linxia, "Implementation and Validation of Aortic Remodeling in Hypertensive Rats" (2014). Mechanical \& Materials Engineering Faculty Publications. 110.

https://digitalcommons.unl.edu/mechengfacpub/110

This Article is brought to you for free and open access by the Mechanical \& Materials Engineering, Department of at DigitalCommons@University of Nebraska - Lincoln. It has been accepted for inclusion in Mechanical \& Materials Engineering Faculty Publications by an authorized administrator of DigitalCommons@University of Nebraska Lincoln. 


\author{
Shijia Zhao \\ Department of Mechanical \& \\ Materials Engineering, \\ University of Nebraska-Lincoln, \\ Lincoln, NE 68588-0656 \\ Linxia Gu ${ }^{1}$ \\ Department of Mechanical \& \\ Materials Engineering, \\ University of Nebraska-Lincoln, \\ Lincoln, NE 68588-0656; \\ Nebraska Center for Materials and Nanoscience, \\ Lincoln, NE 68588-0656 \\ e-mail: Igu2@unl.edu
}

\section{Implementation and Validation of Aortic Remodeling in Hypertensive Rats}

A computational framework was implemented and validated to better understand the hypertensive artery remodeling in both geometric dimensions and material properties. Integrating the stress-modulated remodeling equations into commercial finite element codes allows a better control and visualization of local mechanical parameters. Both arterial thickening and stiffening effects were captured and visualized. An adaptive material remodeling strategy combined with the element birth and death techniques for the geometrical growth were implemented. The numerically predicted remodeling results in terms of the wall thickness, inner diameter, and the ratio of elastin to collagen content of the artery were compared with and fine-tuned by the experimental data from a documented rat model. The influence of time constant on the material remodeling was also evaluated and discussed. In addition, the geometrical growth and material remodeling were isolated to better understand the contributions of each element to the arterial remodeling and their coupling effect. Finally, this framework was applied to an imagebased 3D artery generated from computer tomography to demonstrate its heterogeneous remodeling process. Results suggested that hypertension induced arterial remodeling are quite heterogeneous due to both nonlinear geometry and material adaptation process. The developed computational model provided more insights into the evolutions of morphology and material of the artery, which could complement the discrete experimental data for improving the clinical management of hypertension. The proposed framework could also be extended to study other types of stress-driven tissue remodeling including in-stent restenosis and grafting. [DOI: 10.1115/1.4027939]

Keywords: artery, remodeling, wall stress, tissue mechanics, mechanical stimuli, hypertension

\section{Introduction}

Hypertension, i.e., elevated systolic blood pressure, is a risk factor for many cardiovascular diseases, such as stroke, aneurysm, and heart failure [1]. It is estimated that hypertension will affect approximately $1.56 \times 10^{9}$ people by 2025 [2]. A better risk assessment and treatment requires further understanding of vascular remodeling or adaptation in response to the high blood pressure [3]. Animal studies have been extensively used to assess the influence of high blood pressure on the arterial adaptations including the arterial thickening and stiffening [4-9]. It was believed that these arterial adaptations in terms of geometry and material properties are to restore the biomechanical environment of the arterial wall, i.e., keeping the wall stresses in the range of normotensive conditions [10].

Various theoretical models were developed to better understand the mechanism of arterial remodeling in response to mechanical stimuli including hypertensive pressure. Rachev et al. have proposed geometrical evolution equations regulated by the average circumferential wall stress and/or shear stress [10,11]. The wall stress was calculated by the principle of Laplace's law, and the mean shear stress by the Poiseuille's law. Later they added the material evolution equations regulated by the constituent-based strain energy function to account for the constituents and structural properties of the arterial wall [1,12]. The evolutions of arterial geometry and material properties were captured in terms of the inner diameter, wall thickness, opening angle [11], the mechanical properties of the artery [10] with and without association with the mass fraction of elastin/collagen content [1,12].

\footnotetext{
${ }^{1}$ Corresponding author.

Manuscript received February 19, 2014; final manuscript received June 18, 2014 accepted manuscript posted July 2, 2014; published online July 15, 2014. Assoc. Editor: Dalin Tang
}

However, this series of mathematical models were limited to idealized thin-walled cylindrical arteries due to the average stress estimations.

The local stress/strain calculations are related to the local deformations, whose calculation is time-consuming especially within the complex arterial structure under nonlinear loading conditions. Mathematical modeling of the local stress field was limited to relatively simple geometries. The calculation of layer-specific stress by Rodríguez et al. [13] has been adopted to predict the local growth of the rat aorta in response to the variations of blood pressure from the first heartbeat in the embryo to the maturity, as well as the hypertensive conditions $[14,15]$. With the advancement in finite element method for obtaining the stress field of any 3D geometry, the remodeling framework was enhanced with application to a realistic adult artery $[16,17]$. Local volume or geometry changes were captured. However, the material remodeling was never considered, i.e., the mechanical properties of the arterial tissue keep unaltered during the remodeling process. In addition, for the validation purpose, the existing study of remodeling was mainly compared with the reported evolution history of wall thickness only [16]. A complete validation against a set of experimental data is desired to understand many interacting factors.

The goal of this work is to develop a computational framework implemented in the commercial finite element codes Abaqus (Dassault Systèmes Simulia Corp.), which couples both local geometrical changes and material remodeling for any 3D tissue. The experimental data of a thoracic aorta from both normotensive and hypertensive Wistar-Kyoto rat models [18], including the measured geometry, material properties, and pressure at time points of 3,9 , and 15 months, were used to fine-tune our computational model. Moreover, the roles of isolated geometrical remodeling, material remodeling alone, and the time constant were 
investigated. Finally, we presented an image-based artery to illustrate the versatile features of our computational framework.

\section{Materials and Methods}

Our remodeling framework used high blood pressure as the single input parameter, and predicted the alternations of inner diameter, wall thickness, and arterial stiffness, which were regulated by the remodeling equations described below. The hypertensioninduced intramural stress, specifically the first principal stress, was adopted as the mechanical stimulus.

2.1 Geometrical Remodeling Equation. The governing equation of the geometrical remodeling was adopted and modified from the literature [14], where the growth of aorta along the radial direction was assumed to be correlated with the deviations of the average hoop stress in the arterial wall from its homeostatic state as

$$
\frac{\partial \lambda_{\mathrm{r}}}{\partial t}=\frac{1}{T_{\mathrm{r}}}\left(\frac{\sigma_{\theta}-\sigma_{\theta}^{\mathrm{h}}}{\sigma_{\theta}^{\mathrm{h}}}\right)
$$

where $\lambda_{\mathrm{r}}$ denotes the principal stretch ratio in the radial direction, $\sigma_{\theta}$ as the average hoop stress, $\sigma_{\theta}^{\mathrm{h}}$ as the homeostatic average hoop stress, $t$ as the time, and $T_{\mathrm{r}}$ represents the time constant.

In this work, finite element approach allows us to approximate the partial differential equations locally as algebraic equations. The first principal stress was chosen as the mechanical stimulus. Then the governing equation for geometrical remodeling, specifically the alternations of wall thickness, was simplified as

$$
\frac{\Delta H}{H_{0}}=\frac{1}{T}\left(\frac{\sigma_{1}-\sigma_{1}^{\mathrm{h}}}{\sigma_{1}^{\mathrm{h}}}\right) \Delta t
$$

where $H_{0}$ is the normotensive thickness, $\Delta t$ is the time duration in months, $\Delta H$ is the thickness change after $\Delta t, T$ is the time constant, while $\sigma_{1}$ and $\sigma_{1}^{\mathrm{h}}$ are the first principal stress under hypertensive and normotensive conditions, respectively. The remodeling process will not terminate until the peak first principal stress falls into the target homeostatic stress range, also referred to as a lazy zone.

2.2 Material Remodeling Equation. The ratio of elastin to collagen content $(E / C$ ratio) was chosen to monitor the evolution of material property based on the experimental results from the hypertensive rat models [18]. The incremental changes of the $E / C$ ratio were regulated by the first principal stress, which was consistent with the geometrical remodeling governing equation. The material remodeling process in terms of the $E / C$ ratio will not terminate until the first principal stress at each integration point falls into the lazy zone or reaches the maximum and minimum limits of the $E / C$ ratio. Motivated by the bone remodeling cases [19], the material remodeling scheme, i.e., the $E / C$ ratio variation $(\Delta(E / C))$ in response to the first principal stress at each integration point, was expressed as

$$
\Delta \frac{E}{C}=\left\{\begin{array}{lcc}
\frac{1}{T} * m_{\text {gain }} *\left[\sigma_{1}-(1+S) \sigma_{1}^{\mathrm{h}}\right] * \Delta t & \text { if } & \sigma_{1}>(1+S) \sigma_{1}^{\mathrm{h}} \\
0 & \text { if } \quad & (1-S) \sigma_{1}^{\mathrm{h}} \leq \sigma_{1} \\
& \leq(1+S) \sigma_{1}^{\mathrm{h}} \\
\frac{1}{T} * m_{\text {loss }} *\left[\sigma_{1}-(1-S) \sigma_{1}^{\mathrm{h}}\right] * \Delta t & \text { if } \quad & \sigma_{1}<(1-S) \sigma_{1}^{\mathrm{h}}
\end{array}\right.
$$

where $\mathrm{S}$ denotes the half width of lazy zone, $m_{\text {gain }}$ and $m_{\text {loss }}$ are the gain and loss slopes for regulating the changes in $E / C$ ratio, respectively; $\sigma_{1}, \sigma_{1}^{\mathrm{h}}, \Delta t$, and $T$ are defined the same as Eq. (2). The recommended percentage variation $\pm 10 \%$ was used for the lazy zone [20], i.e., $S=10 \%$. The $E / C$ ratio has a correlation with the elastic modulus of the artery at each integration point, as described in section 2.3.

2.3 Finite Element Modeling. The experimental data of a thoracic aorta from hypertensive Wistar-Kyoto rat models [18] were used to define and fine-tune parameters for our predictive model. The normotensive aorta was considered as a hollow cylinder defined by the average measurements. More specifically, the artery has an inner diameter of $1.38 \mathrm{~mm}$, wall thickness of $0.062 \mathrm{~mm}$, and length of $0.5 \mathrm{~mm}$. It was then meshed into 2516 3D brick elements (C3D8R). The linear elastic material behavior of the aorta was characterized by an initial elastic modulus of $0.7 \mathrm{MPa}$, corresponding to the measured initial $E / C$ ratio of 1.02 , and Poisson's ratio of 0.4 . A transmural pressure of $122 \mathrm{~mm} \mathrm{Hg}$ was applied to the aorta and resulted in a peak first principal stress $\sigma_{1}^{\mathrm{h}}=0.1855 \mathrm{MPa}$ on the arterial wall, which was taken as the homeostatic stress state. The model computed value agrees with the average wall stress of $0.18 \pm 0.01 \mathrm{MPa}$ calculated from the experiments. All these input parameters were the average values estimated from experiments.

The hypertension-induced remodeling progress was initiated by applying an elevated pressure of $172 \mathrm{~mm} \mathrm{Hg}$. The remodeling time step for both geometrical and material remodeling was set as $\Delta t=1$ month $=720 \mathrm{~h}$ and the time constant as $T=4000 \mathrm{~h}$. The relationship between the $E / C$ ratio and elastic modulus of aorta, adopted from the experimental data [18], was listed in Table 1. A linear interpolation was used between data points. The upper and lower bounds for the $E / C$ ratio were set as 1.88 and 1.02 , respectively. The gain slope was estimated by substituting the related experimental data into Eq. (3) as

$$
m_{\text {gain }}=\left\{\begin{array}{lll}
78 & \text { if } & 0 \leq i \leq 3 \\
11 & \text { if } & 3<i \leq 9 \\
37 & \text { if } & 9<i
\end{array}\right.
$$

where $\mathrm{i}$ is the time in month. The loss slope was chosen as $m_{\mathrm{loss}}=3$, while the first principal stress range of $0.1855 \pm 0.01855 \mathrm{MPa}$ was set as the lazy zone based on $S=10 \%$.

The governing equations for material remodeling were implemented using a user subroutine USDFLD coded in FORTRAN programming language. The $E / C$ ratio, defined as one field variable, was updated on integration points at each time increment. The new arterial stiffness, corresponding to a new $E / C$ ratio, was estimated as a linear interpolation between data points defined in Table 1 . The stress results at each time step, i.e., 1 month, were extracted from the output database, and then used to regulate the geometrical remodeling. A Python script was developed to implement the governing equation for geometrical remodeling, update the local geometry changes at each step using the element birth and death techniques, incorporate the material remodeling subroutine, and enforce the termination criteria for the remodeling process.

As the hypertensive pressure is applied to the artery specified above, the resulted wall stress is much larger than its homeostatic value, which then activates both the geometrical and material remodeling process, i.e., the arterial wall thickening and stiffening. The remodeling will continue until first principal stresses falls

Table 1 The correlation between the E/C ratio and the elastic modulus of aorta

\begin{tabular}{lllll}
\hline \hline$E / C$ ratio & 1.02 & 1.32 & 1.62 & 1.88 \\
Elastic modulus (MPa) & 0.7 & 1.6 & 0.9 & 1.2 \\
\hline \hline
\end{tabular}


into the targeted stress range as defined above. The predicted wall thickness, inner diameter, elastic modulus, and wall stress at various time points were compared with the experimental data. In addition, the proposed computational framework could be used for any $3 \mathrm{D}$ geometries with heterogeneous material properties. An image-based artery reconstructed from computer tomography (CT) was then served as a demonstrative case.

\section{Results}

3.1 Validated and Fine-Tuned Aortic Remodeling in a Rat Model. Figure 1 has compared the evolution histories of the peak first principal stress, wall thickness, inner diameter, and elastic modulus of aorta obtained from our computational model with the published experimental data [18]. Data at the 0 month represented the normotensive state. Generally speaking, our model captured the remodeling progress in terms of material evolutions coupled with the geometrical ones. Extra remodeling information beyond the discrete experimental data points at the $0,3,9$, and 15 months was revealed by the computational model. For example, the first principal stress, as the mechanical stimulus modulating the remodeling process, demonstrated a sharp elevation at the first month of the remodeling, as shown in Fig. 1(a). Then this stress gradually decreased and matched very well with the fragmented experimental data points. This sharp change, however, was only observed in our modeling results. At the 15 th month, the peak first principal stress was $0.2031 \mathrm{MPa}$, which fell into the targeted stress range of $0.1855 \pm 0.01855 \mathrm{MPa}$. The remodeling process was then terminated.

The evolution of wall thickness from our model showed a continuously smooth wall thickening process in response to hypertension (Fig. 1(b)). It predicted that the thickness of aortic wall increased from normotensive value of $0.062 \mathrm{~mm}$ to $0.074 \mathrm{~mm}$, $0.088 \mathrm{~mm}$ and $0.095 \mathrm{~mm}$ at 3,9 , and 15 months of hypertension, respectively. The reported experimental data were 0.081 $\pm 0.006 \mathrm{~mm}, 0.080 \pm 0.004 \mathrm{~mm}$, and $0.095 \pm 0.004 \mathrm{~mm}$ at the 3 , 9 , and 15 months of hypertension, respectively. A plateau in the experimental data was observed during the period from 3 to 9 months. It is interesting to see that the wall thickness remodeling converged at the same value at the 15 months of hypertension.

The inner diameters of aorta after 3,9 , and 15 months of remodeling were predicted as $1.63,1.61$, and $1.60 \mathrm{~mm}$, respectively. The corresponding experimental measurements were reported as $1.61 \pm 0.07 \mathrm{~mm}, 1.55 \pm 0.06 \mathrm{~mm}$ and $1.78 \pm 0.10 \mathrm{~mm}$, respectively. Both experiments and simulation demonstrated a gradually reduced lumen up to the 9 th month (Fig. 1(c)). A notable discrepancy was observed at the 15th month of remodeling. Experimental results showed a substantial increase in the inner diameter, which is not the case for the simulation. This discrepancy was observed once again at the same time point for the evolution of the elastic moduli of aorta. It is clear that the modeling captured the evolution of elastic moduli up to the 9th month (Fig. 1(d)). The experimentally observed elastic modulus at the 15 th month, i.e., $1.6 \pm 0.2 \mathrm{MPa}$, was far above the predicted value of 1.02 MPa. The discrepancy could be attributed to the ageing effect

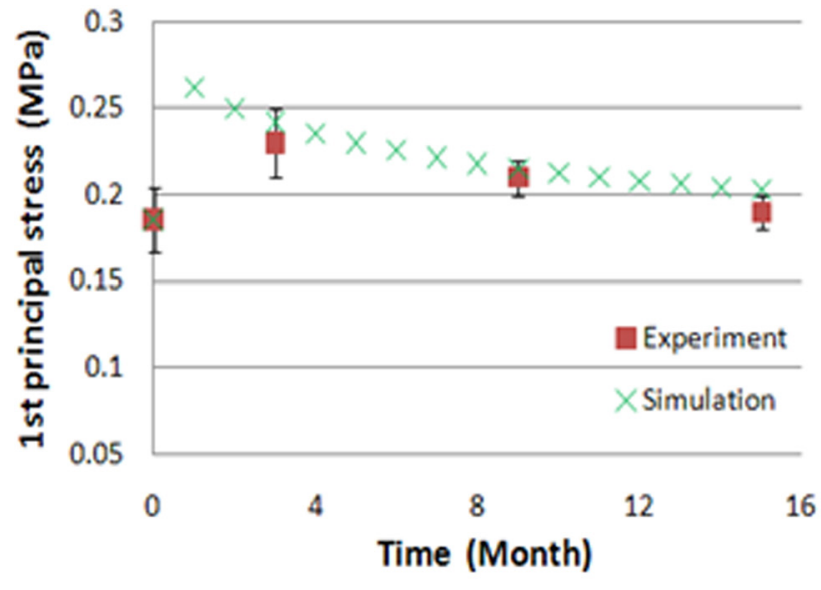

(a)

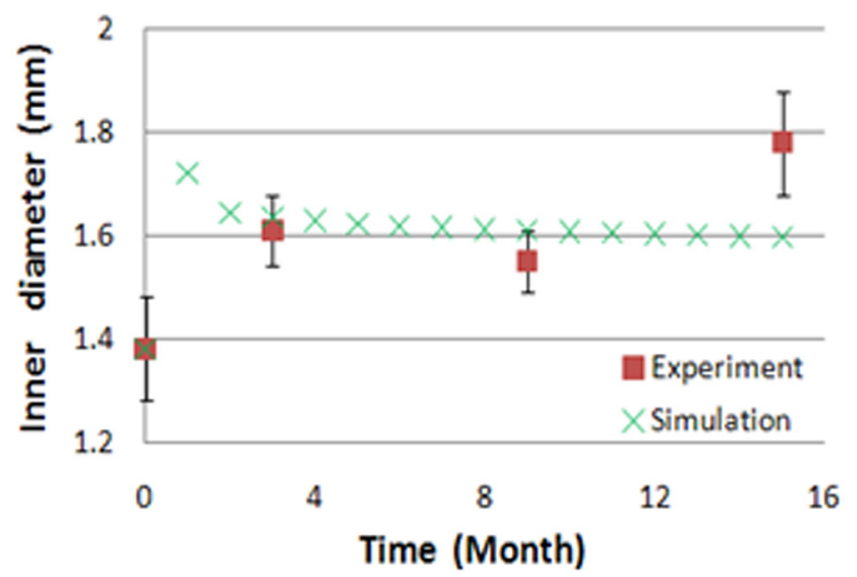

(c)

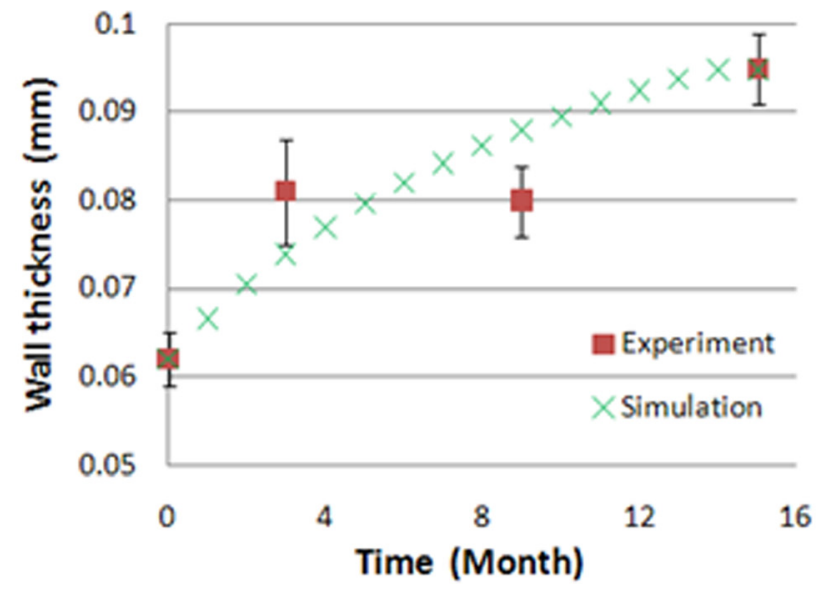

(b)

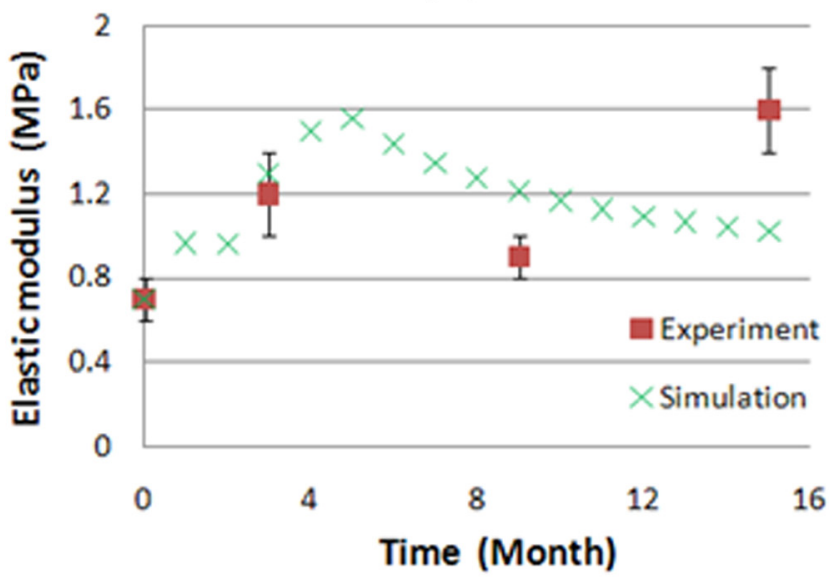

(d)

Fig. 1 The evolution history comparisons between experiment and simulation: (a) peak first principal stress, (b) wall thickness, (c) inner diameter, and (d) elastic modulus of aorta 
of the rat as well as the assumed linear interpolation relationship between the $E / C$ ratio and the elastic modulus, which will be discussed later.

3.2 The Role of Time Constant. To assess the role of time constant on the remodeling process, we altered the time constant for material remodeling from $4000 \mathrm{~h}$ to 18,000 and $40,000 \mathrm{~h}$, while keeping the time constant for geometrical remodeling unchanged [1,12]. Figure 2 depicted the comparative results between these three cases in terms of peak first principal stress, wall thickness, inner diameter, and elastic modulus. It is clear that a larger time constant in the new model, i.e., the increased time constant for the material remodeling, resulted in a slower remodeling process. The remodeling process with a relatively larger time constant took a few more months, specifically 16 months for the case with time constant of $18,000 \mathrm{~h}$ and 19 months for the case with time constant of $40,000 \mathrm{~h}$, to get back to the homeostatic stress state. Moreover, it induced a larger arterial wall thickening, which was backed up by the evidence of a relatively larger peak wall stress in the new model. This wall thickening led to a relatively smaller elastic modulus of arterial wall as well as the almost unchanged lumen size.

3.3 The Effect of Isolated Geometrical Remodeling. To understand the role of geometrical remodeling on the arterial adaptations, one case considering the geometrical remodeling only was presented and compared to our base model, which coupled the effects of both geometrical and material remodeling. Figure 3 has depicted the evolution of peak first principal stress, arterial wall thickness, and inner diameter for both cases. The profound differences between these two cases were the fast remodeling process and the larger lumen for the case with geometrical remodeling only. The peak first principal stress, the modulator of arterial remodeling, fell into the lazy zone in 9 months period compared to the 15 months used for the base model. Without the arterial stiffening, it is obvious that the inner diameter of the artery is increased dramatically. A relatively thinner wall thickness was also observed. Both of these geometrical changes were integrated for alleviating the elevated arterial stresses. It is clear that the arterial stiffening combined with the increased wall thickness of the artery contributed to sustaining the elevated blood pressure. The geometrical remodeling alone is capable of regulating the arterial stress, but it could underestimate the evolution of arterial thickness without considering arterial stiffening.

3.4 The Effect of Isolated Material Remodeling. One case considering the material remodeling only was presented here and compared to our base model to illustrate the role of arterial stiffening under hypertension, as shown in Fig. 4. The interdependency between first principal stress and arterial stiffness made it difficult or impossible in this case to bring the arterial stress back to the homeostatic state. It is obvious that the artery was stiffened in the first 2 months of remodeling to bring down a little bit of peak first principal stress. Then it stabilized at certain stress and stiffness level. This large stiffness level also led to a reduced lumen size. The material remodeling alone demonstrated a deficient regulation of arterial wall back to its homeostatic state. It indicated that the arterial wall thickening is essential to regulate the stress state. However, the material remodeling scheme did complement the

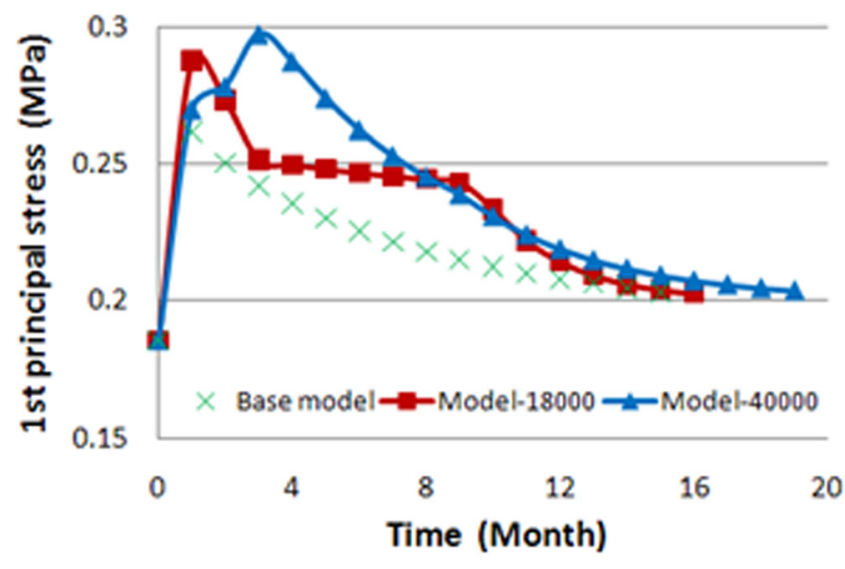

(a)

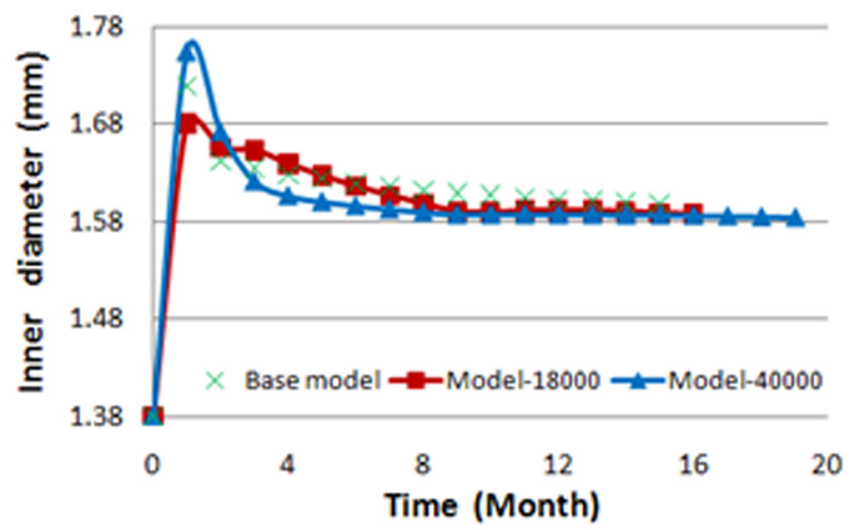

(c)

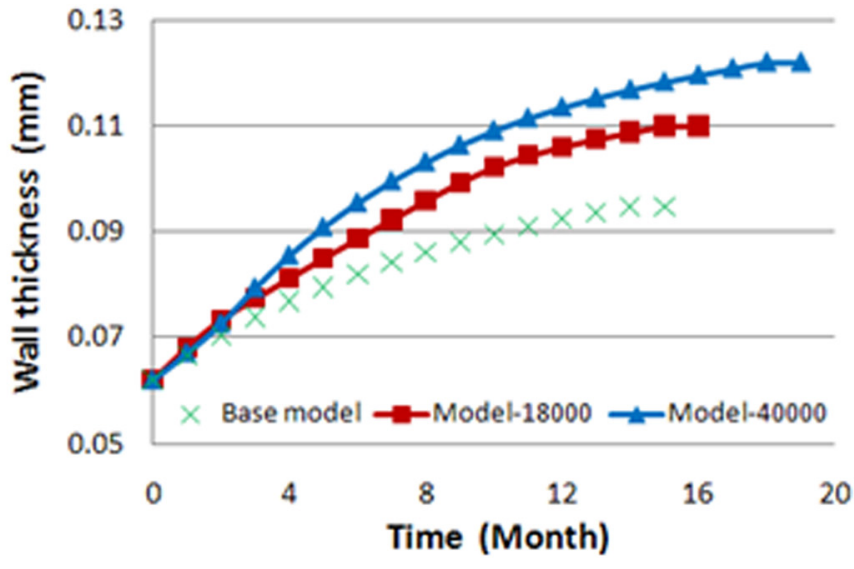

(b)

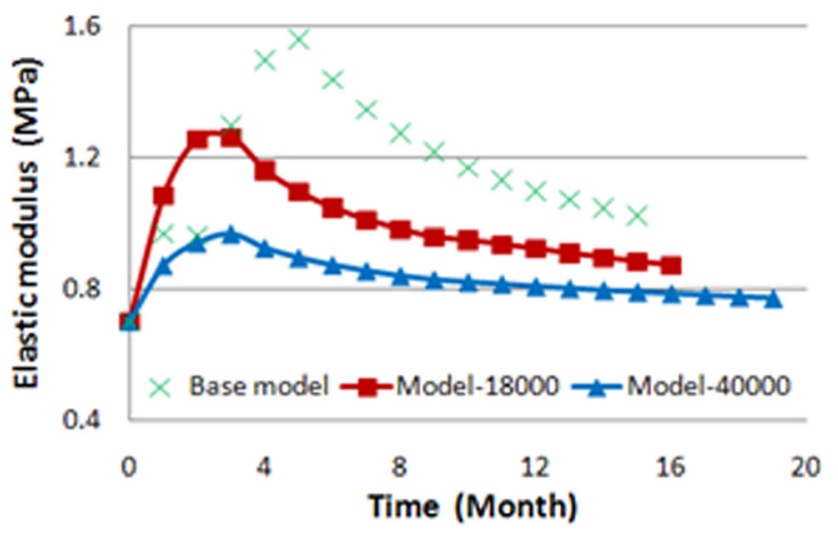

(d)

Fig. 2 The role of time constant: (a) peak first principal stress, (b) wall thickness, (c) inner diameter, and (d) elastic modulus of aorta 


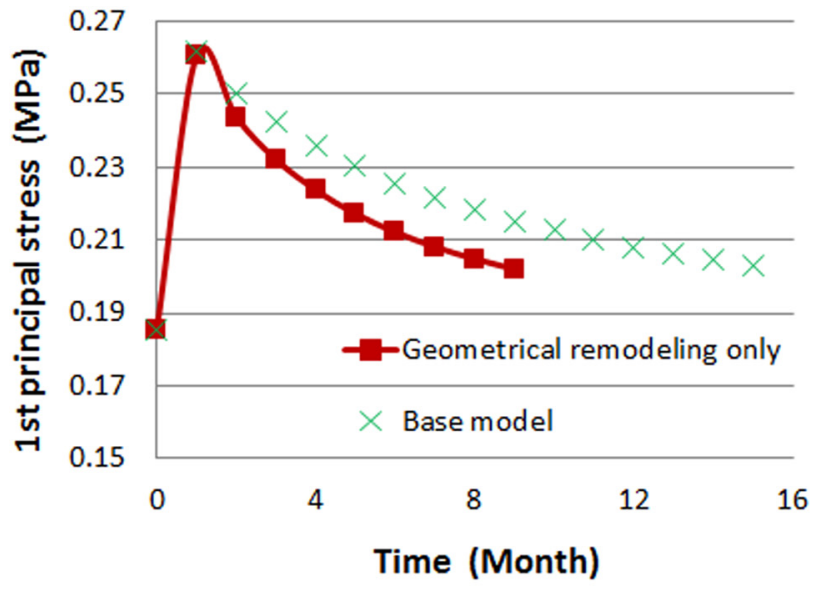

(a)

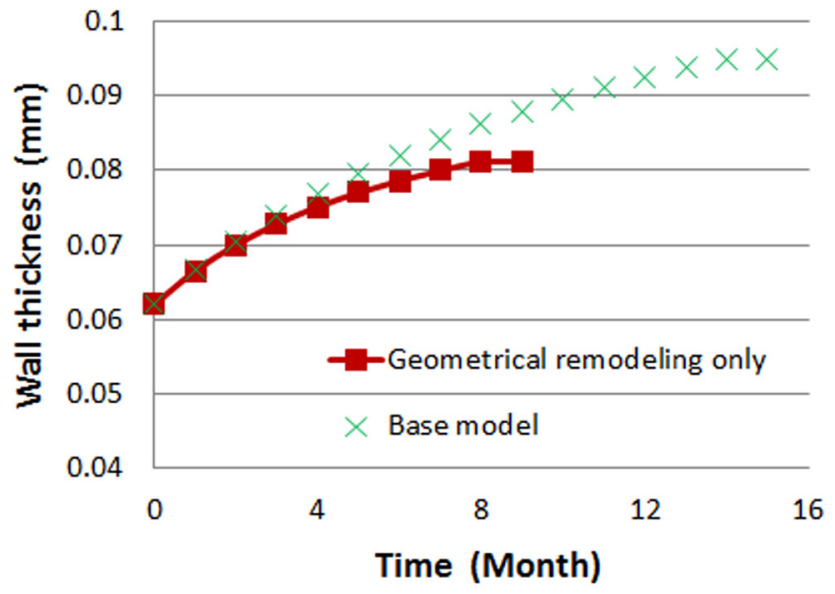

(b)

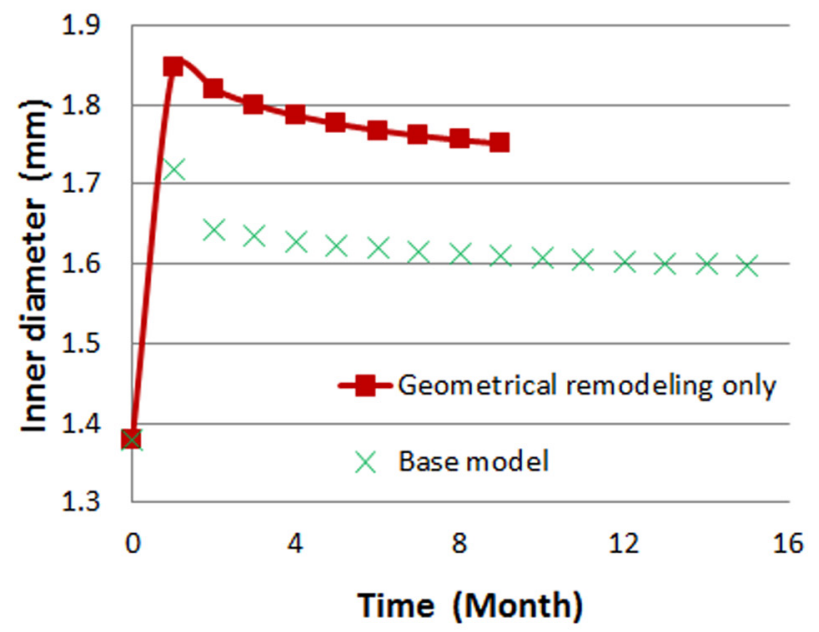

(c)

Fig. 3 Cases with and without material remodeling: (a) peak first principal stress, (b) wall thickness, and (c) inner diameter

geometrical remodeling to capture the reported arterial thickening and stiffening.

3.5 Image-Based Artery Remodeling Under Hypertension. The validated computational remodeling framework was applied to an image-based artery to demonstrate its generality. A right carotid artery reconstructed from CT images was discretized into 30,528 brick elements, as shown in Fig. 5. The hypertension pressure of $160 \mathrm{~mm} \mathrm{Hg}$ was applied to initiate the remodeling. The inhomogeneous neo-tissue growth and material remodeling were illustrated in Fig. 6 for two random chosen cross sections labeled as $m-m$ and $n-n$.

Before remodeling, the elevated arterial stress under hypertension is heterogeneous with different patterns at various cross sections (left figures). At this point, the material composition of the artery is homogenous with the constant $E / C$ ratio of 1.02. The abnormal stresses led to a thicker arterial wall (middle figures) especially at locations with higher stresses. The nonuniform growth was highlighted at the outer layer of the hypertensive artery. The percentage of reduction in lumen size at cross section $m-m$ was $12.65 \%$ along the straight line 11 , and $10.56 \%$ along the line 2-2, respectively. At cross section $n-n$, it was $11.59 \%$ along the line $3-3$, and $6.45 \%$ along the line $4-4$, respectively. It is obvious that the material remodeling of the hypertensive artery is also inhomogeneous as illustrated by the $E / C$ ratio maps, labeled as SDV3 (right figures). The artery is evolved from the homogenous $E / C$ ratio of 1.02 to the heterogeneous range of 0.71 to 2.19 under hypertension. Again we observed that the hypertension induced arterial wall thickening and stiffening with profound heterogeneity.

\section{Discussions}

Arteries are sensitive to alternations in their biomechanical environment. It is well acknowledged that artery changes its geometry and composition of tissue, also referred to as remodeling, for regaining the homeostatic state $[21,22]$. In the perspective of mechanics, the remodeling process is regulated by mechanical parameters, such as the stress [12,23], strain [24], or strain energy density [25]. In this work, we have proposed a computational framework for better describing the arterial remodeling mechanics in response to hypertension. Our model is capable of coupling both geometrical and material remodeling, as well as handling any $3 \mathrm{D}$ geometry and heterogeneous materials. The peak first principal stress was chosen to modulate the remodeling process. The governing equation for the stress-driven material remodeling was motivated from the bone remodeling [19] and the one for geometrical remodeling was adopted from Taber's work [14]. The proposed remodeling scheme has been implemented into the commercial finite element codes using a user subroutine USDFLD and element birth/death techniques. It was validated against the published hypertensive data of a thoracic aorta in Wistar-Kyoto rat models [18]. The experimental data was also used to estimate the parameters in the remodeling governing equations.

Both the arterial wall thickening and stiffening in response to hypertension were well captured through our computational model. The remodeling process indeed brought the peak first principal stress back to the homeostatic stress ranges, i.e., lazy zone. The computed wall thickness evolution matched well with the 


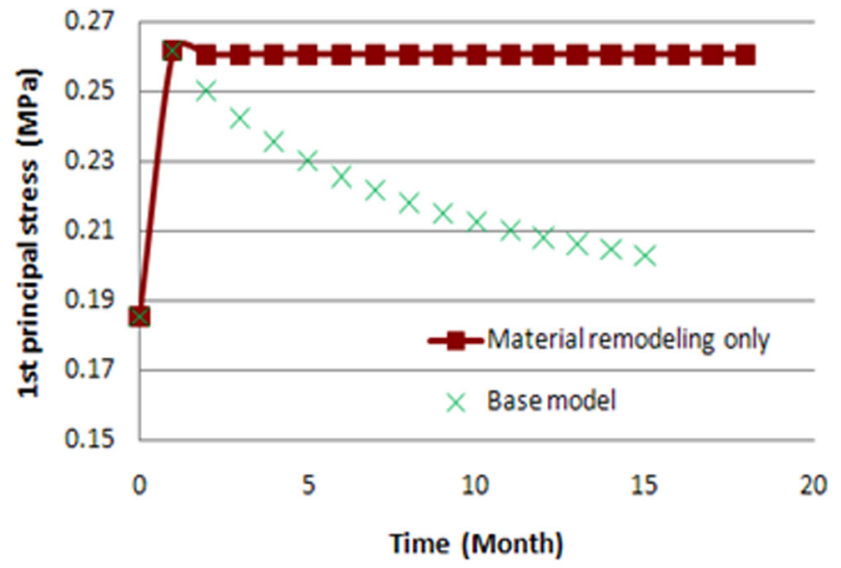

(a)

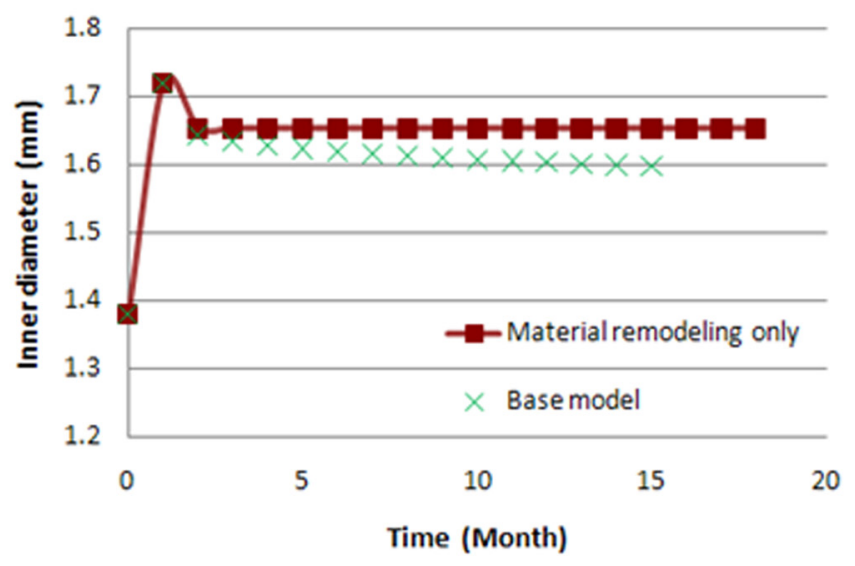

(b)

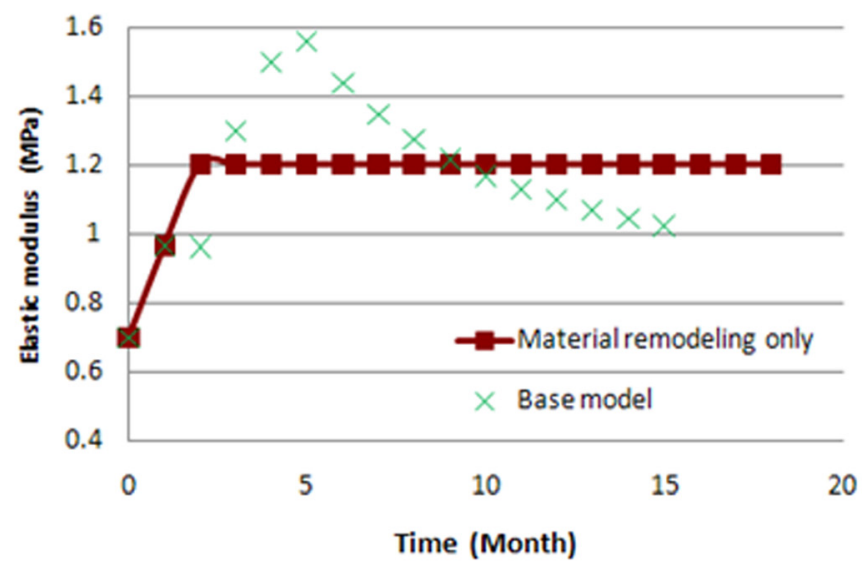

(c)

Fig. 4 Cases with and without geometry growth: (a) peak first principal stress, (b) inner diameter, and (c) elastic modulus

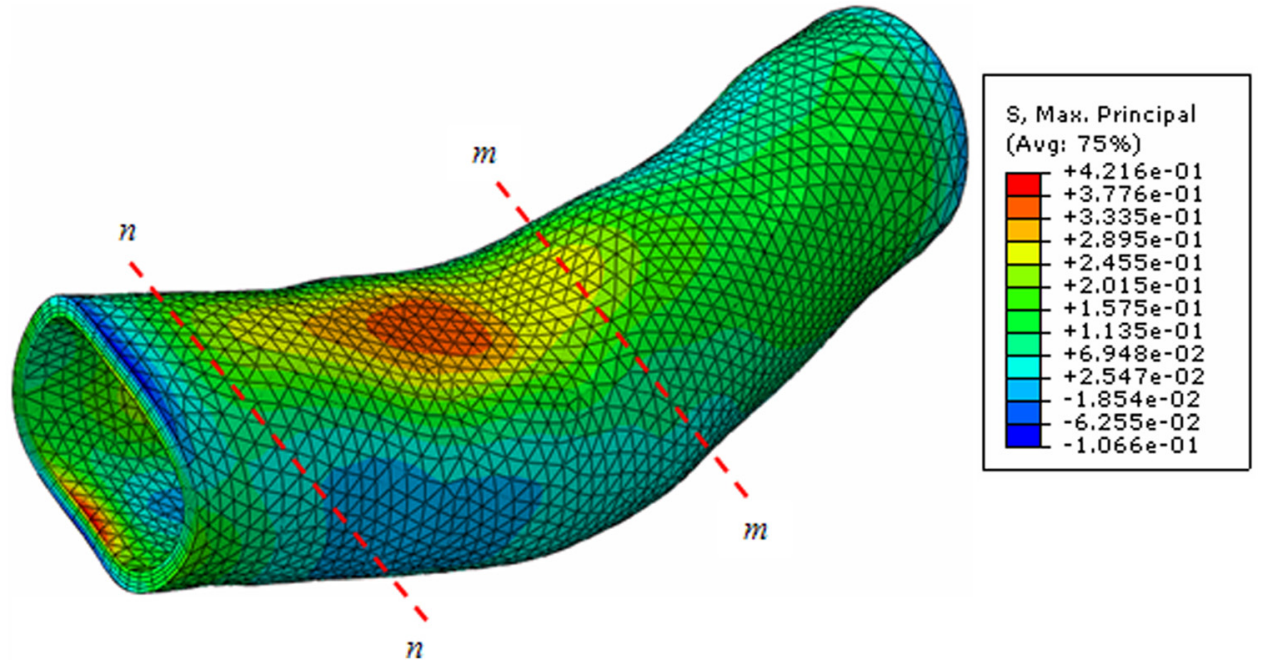

Fig. 5 Stress distribution of an image-based artery

measured ones. The predictability of our computational model was challenged for the inner diameter and elastic modulus at the 15 th month of remodeling only (Fig. 1). Considering that 15 months of rat age is equivalent to $37 \mathrm{yr}$ of human ages [26], the aging effect, specifically increased stiffness of artery, could play a part in the arterial remodeling. We experimented with a larger initial $E / C$ ratio of 1.32 versus original 1.02 at the beginning of the remodeling process. The evolution of elastic modulus appeared as a concave shape opposite from the convex curve in our original base model. This could be used to explain the observed sharp increase in stiffness at the 15th month. It also indicated that both aging and elevated pressure led to arterial stiffening, resulting in a significant increase in the elastic modulus at the later stage of remodeling. To capture and incorporate the aging effect into the remodeling framework for better prediction of the material property change in artery, more experimental data points are needed to calibrate the parameters in governing equations. 

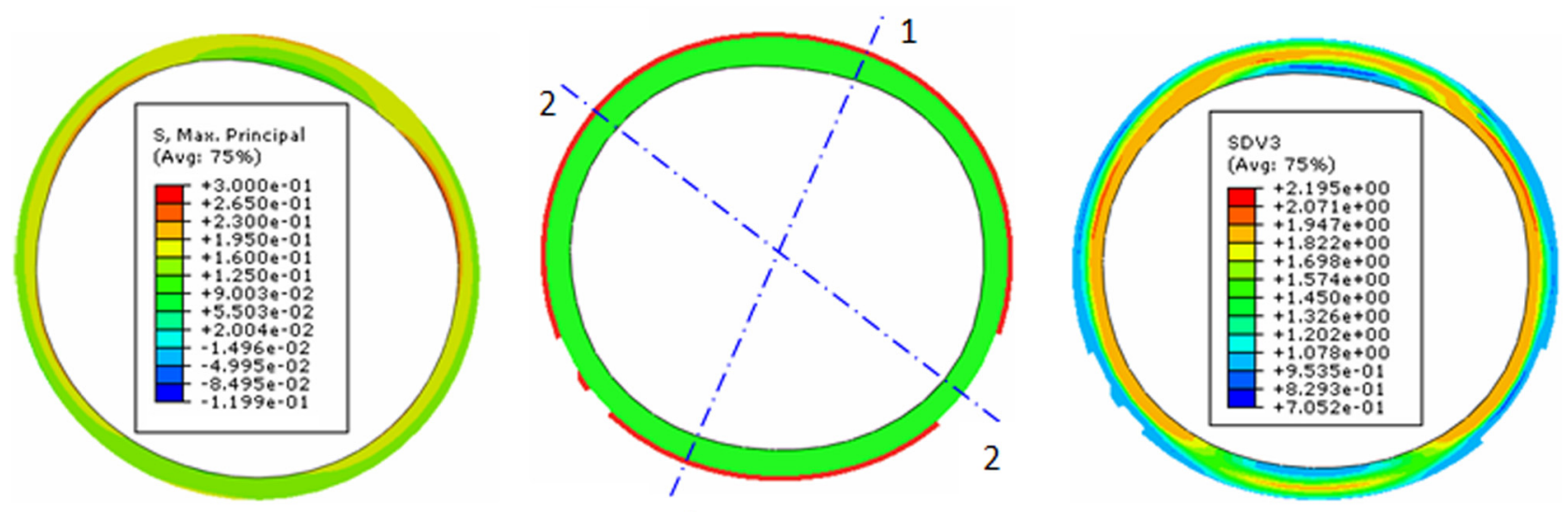

1
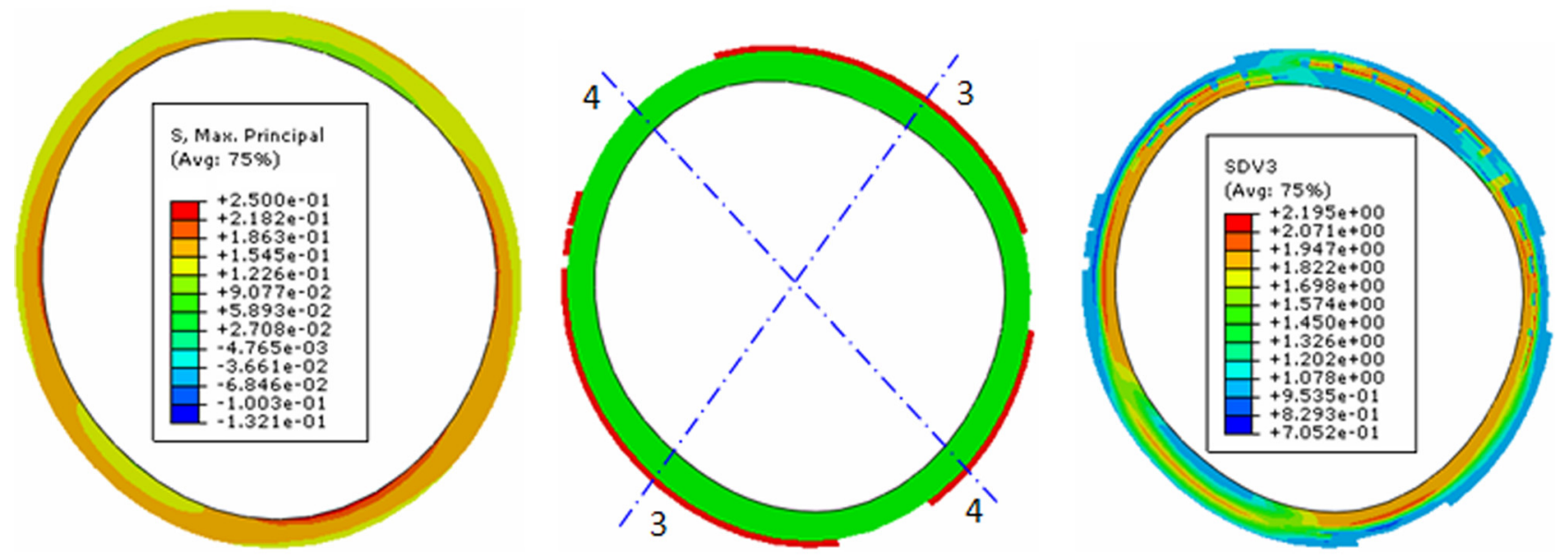

(b)

Fig. 6 Heterogeneous geometrical and material remodeling of an image-based artery at (a) cross section $m-m$ and $(b)$ cross section $n$ - $n$. Left: maximum principal stress map before remodeling; middle: highlighted neo-tissue after remodeling; right: the $E / C$ ratio map.

The roles of geometry growth and stiffness change were isolated to better understand the contribution from each element to the arterial remodeling. The geometrical remodeling alone has demonstrated its capacity to regulate the arterial stress back to its homeostatic state, which was well demonstrated in the existing literature. Lack of arterial stiffening effect resulted in a larger lumen size and a thinner wall of the artery to compensate for the elevated pressure. This indicated that the geometrical remodeling alone might underestimate the evolution of arterial thickness. In contrast, the material remodeling alone has shown its inability to regulate the arterial wall stresses back to its homeostatic state. This could be explained by the tight interdependence between arterial stress and its stiffness. The arterial stiffening alone generally led to a higher arterial stresses and a decreased lumen size, which is not enough to alleviate the hypertension-induced arterial stress. However, it did complement the geometrical remodeling to better capture the remodeling process as evidenced in the literature [27].

The simultaneous remodeling in terms of both geometrical growth and material adaptations was assumed in our computational model. This might not hold true for any type of hypertension. For example, previous rat studies have shown that the dimensional changes precede material property adaptation to hypertension, i.e., material remodeling is slower than geometry growth [8]. The time constant in the governing equations is the major regulator of the remodeling speed. The impact of time constant on the arterial remodeling was investigated by changing the time constant for material remodeling from 4000 to 18,000 or $40,000 \mathrm{~h}$. This way the material remodeling is slower than the geometrical remodeling. The computational results showed that the increased time constant for the material remodeling delayed the arterial stiffening and resulted in a higher arterial stress, which required a thicker artery wall to regain its homeostatic state. Appropriate experiments need to be designed to estimate reliable time constants.

Our computational framework has also demonstrated its capacity in capturing the heterogeneous remodeling process of one image-based artery. The $3 \mathrm{D}$ geometry of the artery was reconstructed from a stack of CT images. The nonlinearity of the artery geometry led to complex stress distributions on the arterial wall in response to hypertension. This, in turn, led to the nonuniform remodeling in terms of neo-tissue and the $E / C$ ratio. It was clearly observed that hypertension induced arterial wall thickening and stiffening with evident heterogeneity.

The arterial stretch, radial stress, and wall shear stress were also speculated to contribute to the arterial remodeling [28-30]. Their roles on the arterial remodeling were not explicitly included in this work due to lack of experimental data. The discrete experimental data could be enhanced with more time points as well as more measurements such as the axial stretch, shear stress, residual stress, etc. The current computational framework was also ready to adapt to new mechanical environments by mimicking the remodeling process in the case of stenting, ageing, and grafting 
for better inspecting the underlying mechanobiological processes and enhancing the surgical management of vascular diseases.

\section{Acknowledgment}

This project was partially funded by the National Science Foundation under Grant No. 1254095 and UNL Research Council Interdisciplinary Grant.

\section{References}

[1] Tsamis, A., Stergiopulos, N., and Rachev, A., 2009, "A Structure-Based Model of Arterial Remodeling in Response to Sustained Hypertension," ASME J. Biomech. Eng., 131(10), p. 101004.

[2] Kearney, P. M., Whelton, M., Reynolds, K., Muntner, P., Whelton, P. K., and He, J., 2005, "Global Burden of Hypertension: Analysis of Worldwide Data," Lancet, 365(9455), pp. 217-223.

[3] Taber, L. A., 1995, "Biomechanics of Growth, Remodeling, and Morphogenesis," ASME Appl. Mech. Rev., 48(8), pp. 487-545.

[4] Wolinsky, H., 1970, "Response of the Rat Aortic Media to Hypertension. Morphological and Chemical Studies," Circ. Res., 26(4), pp. 507-522.

[5] Wolinsky, H., 1971, "Effects of Hypertension and Its Reversal on the Thoracic Aorta of Male and Female Rats. Morphological and Chemical Studies," Circ. Res., 28(6), pp. 622-637.

[6] Wolinsky, H., 1972, "Long-Term Effects of Hypertension on the Rat Aortic Wall and Their Relation to Concurrent Aging Changes. Morphological and Chemical Studies," Circ. Res., 30(3), pp. 301-309.

[7] Liu, S. Q., and Fung, Y. C., 1989, "Relationship Between Hypertension, Hypertrophy, and Opening Angle of Zero-Stress State of Arteries Following Aortic Constriction," ASME J. Biomech. Eng., 111(4), pp. 325-335.

[8] Matsumoto, T., and Hayashi, K., 1994, "Mechanical and Dimensional Adaptation of Rat Aorta to Hypertension,” ASME J. Biomech. Eng., 116(3), pp. 278-283.

[9] Vaishnav, R. N., Vossoughi, J., Patel, D. J., Cothran, L. N., Coleman, B. R., and Ison-Franklin, E. L., 1990, "Effect of Hypertension on Elasticity and Geometry of Aortic Tissue From Dogs," ASME J. Biomech. Eng., 112(1), pp. 70-74.

[10] Rachev, A., Stergiopulos, N., and Meister, J. J., 1998, "A Model for Geometric and Mechanical Adaptation of Arteries to Sustained Hypertension," ASME J. Biomech. Eng., 120(1), pp. 9-17.

[11] Rachev, A., Stergiopulos, N., and Meister, J. J., 1996, "Theoretical Study of Dynamics of Arterial Wall Remodeling in Response to Changes in Blood Pressure," J. Biomech., 29(5), pp. 635-642.

[12] Tsamis, A., and Stergiopulos, N., 2007, “Arterial Remodeling in Response to Hypertension Using a Constituent-Based Model," Am. J. Physiol.-Heart C, 293(5), pp. H3130-H3139.

[13] Rodríguez, E. K., Hoger, A., and McCulloch, A. D., 1994, "Stress-Dependent Finite Growth in Soft Elastic Tissues," J. Biomech., 27(4), pp. 455-467.
[14] Taber, L. A., 1998, "A model for Aortic Growth Based on Fluid Shear and Fiber Stresses,” ASME J. Biomech. Eng., 120(3), pp. 348-354.

[15] Taber, L. A., and Humphrey, J. D., 2001, "Stress-Modulated Growth, Residual Stress, and Vascular Heterogeneity," ASME J. Biomech. Eng., 123(6), pp. $528-535$.

[16] Rodríguez, J., Goicolea, J. M., and Gabaldon, F., 2007, “A Volumetric Model for Growth of Arterial Walls With Arbitrary Geometry and Loads," J. Biomech., 40(5), pp. 961-971.

[17] Alastrue, V., Martinez, M. A., and Doblare, M., 2008, "Modelling Adaptative Volumetric Finite Growth in Patient-Specific Residually Stressed Arteries," J. Biomech., 41(8), pp. 1773-1781.

[18] Marque, V., Kieffer, P., Atkinson, J., and Lartaud-Idjouadiene, I., 1999 , "Elastic Properties and Composition of the Aortic Wall in Old Spontaneously Hypertensive Rats," Hypertension, 34(3), pp. 415-422.

[19] Carter, D. R., 1984, "Mechanical Loading Histories and Cortical Bone Remodeling,” Calcif. Tissue Int., 36(Suppl. 1), pp. S19-24.

[20] Fung, Y. C., 1993, Biomechanics: Mechanical Properties of Living Tissues, Springer-Verlag, New York.

[21] Fung, Y. C., 1990, Biomechanics: Motion, Flow, Stress, and Growth, SpringerVerlag, New York.

[22] Rachev, A., and Greenwald, S. E., 2003, "Residual Strains in Conduit Arteries," J. Biomech., 36(5), pp. 661-670.

[23] Qiu, H. Y., Valtier, B., Struyker-Boudier, H. A., and Levy, B. I., 1995 "Mechanical and Contractile Properties of In Situ Localized Mesenteric Arteries in Normotensive and Spontaneously Hypertensive Rats," J. Pharmacol. Toxicol. Methods, 33(3), pp. 159-170.

[24] Fernandez, J. R., Garcia-Aznar, J. M., Martinez, R., and Viano, J. M., 2010, "Numerical Analysis of a Strain-Adaptive Bone Remodelling Problem," Comput. Methods Appl. Mech. Eng., 199(23-24), pp. 1549-1557.

[25] Huiskes, R., Weinans, H., Grootenboer, H. J., Dalstra, M., Fudala, B., and Slooff, T. J., 1987, "Adaptive Bone-Remodeling Theory Applied to ProstheticDesign Analysis," J. Biomech., 20(11-12), pp. 1135-1150.

[26] Andreollo, N. A., Santos, E. F., Araujo, M. R., and Lopes, L. R., 2012, "Rat's Age Versus Human's Age: What is the Relationship?," Arq. Bras. Cir. Dig., 25(1), pp. 49-51.

[27] Franklin, S. S., 2005, "Arterial Stiffness and Hypertension: a Two-Way Street?," Hypertension, 45(3), pp. 349-351.

[28] Abdel-Khalek, M. A., El-Barbary, A. M., Essa, S. A., and Ghobashi, A. S. 2011, "Serum Hepcidin: a Direct Link Between Anemia of Inflammation and Coronary Artery Atherosclerosis in Patients With Rheumatoid Arthritis," J. Rheumatol., 38(10), pp. 2153-2159.

[29] Fridez, P., Makino, A., Miyazaki, H., Meister, J. J., Hayashi, K., and Stergiopulos, N., 2001, "Short-Term Biomechanical Adaptation of the Rat Carotid to Acute Hypertension: Contribution of Smooth Muscle," Ann. Biomed. Eng., 29(1), pp. 26-34.

[30] Zarins, C. K., Zatina, M. A., Giddens, D. P., Ku, D. N., and Glagov, S., 1987, "Shear Stress Regulation of Artery Lumen Diameter in Experimental Atherogenesis," J. Vasc. Surg., 5(3), pp. 413-420. 